\title{
Successful Chemoradiotherapy for Undifferentiated Malignant Neoplasm Arising from the Left Pulmonary Artery
}

\author{
Hozumi Kumagai $^{a}$ Kenta Nio ${ }^{a}$ Yuta Okumura ${ }^{a}$ Masato Komoda ${ }^{a}$ \\ Tsuyoshi Shirakawa $^{a}$ Hitoshi Kusaba $^{a}$ Shioto Yasuda ${ }^{a}$ Keita Odashiro $^{a}$ \\ Shuji Arita ${ }^{a}$ Hiroshi Ariyama $^{a}$ Yuichi Yamada $^{\text {b }}$ Hidetaka Yamamoto $^{b}$ \\ Yoshinao Oda $^{b}$ Katsumasa Nakamura ${ }^{d}$ Koichi Akashi ${ }^{a}$ Eishi Baba ${ }^{c}$ \\ Departments of ${ }^{\mathrm{a}}$ Medicine and Biosystemic Sciences and ${ }^{\mathrm{b}}$ Anatomic Pathology, \\ Pathological Sciences, Graduate School of Medical Sciences, and 'Department of \\ Comprehensive Clinical Oncology, Faculty of Medical Sciences, Kyushu University, and \\ ${ }^{\mathrm{d}}$ Department of Radiology, Kyushu University Hospital, Fukuoka, Japan
}

\section{Key Words}

Undifferentiated malignant neoplasm · Pulmonary artery tumor · Chemotherapy · Radiotherapy

\begin{abstract}
Undifferentiated malignant neoplasms, which occur primarily in the pulmonary artery, are extremely rare and associated with poor outcomes as there is no effective therapy. A 67year-old woman visited our hospital with complaints of dry cough and dyspnea on exertion. A contrast-enhanced chest computed tomography revealed an intravascular tumor obstructing the left pulmonary artery and a pedunculated lesion extending to the main and right pulmonary artery. Multiple metastases in the lung, bones and bilateral adrenal glands were identified by fluorodeoxyglucose-positron emission tomography. A small sample was obtained by catheter aspiration biopsy of the intravascular tumor, and examination revealed undifferentiated small atypical cells. The tumor was diagnosed as an undifferentiated neoplasm arising from the pulmonary artery based on immunohistochemical findings, including the absence of expressions of organ-specific markers. Systemic chemotherapy (paclitaxel and carboplatin) and concurrent radiation were performed as treatment for the primary tumor. Marked shrinkage of the intravascular tumor was achieved, and no serious adverse events were observed during therapy. Chemotherapy was continued for 5 months,
\end{abstract}

Eishi Baba, MD, PhD

Department of Comprehensive Clinical Oncology

Faculty of Medical Sciences, Kyushu University

3-1-1 Maidashi, Higashi-ku, Fukuoka 812-8582 (Japan)

E-Mail e-baba@c-oncology.med.kyushu-u.ac.jp 
Kumagai et al.: Successful Chemoradiotherapy for Undifferentiated Malignant Neoplasm Arising from the Left Pulmonary Artery

but the patient died because of tumor progression 9 months after the initial diagnosis. Chemoradiotherapy has efficacy against undifferentiated neoplasm of the pulmonary artery.

(c) 2014 S. Karger AG, Basel

\section{Introduction}

Malignant neoplasms arising primarily from the heart and large vessels are rare, and those arising from the pulmonary artery are even rarer. Patients with pulmonary artery tumor are at increased risk of life-threatening events when they become symptomatic. Major artery thromboembolism and acute right heart failure have been frequently observed in such patients [1]. Historically, pulmonary artery tumors were most often diagnosed at autopsy, but recent advances in imaging technology have allowed better antemortem diagnosis. Precise pathodiagnosis of pulmonary artery tumors is difficult because of the necessity to obtain a tissue sample from this hard-to-access lesion. According to past reports, most tumors arising from pulmonary artery were sarcomas including various histologic types, such as undifferentiated sarcoma, leiomyosarcoma, rhabdomyosarcoma and fibrosarcoma [2]. In addition, secondary involvements of pulmonary arteries by lung cancer and pulmonary tumoral embolism caused by various malignancies have been described [2]. Therapeutic surgical resection decreased the clinical symptoms and increased the survival in these patients [3]. Patients who were not suitable candidates for invasive surgery tended to have poor outcomes because effective alternative therapeutic methods, including systemic chemotherapy and radiotherapy, were not well established. In the present report, a very rare case of undifferentiated malignant neoplasm of the pulmonary artery was successfully treated with continuous outpatient chemoradiotherapy without severe cardiovascular events.

\section{Case Presentation}

A 67-year-old Japanese woman was admitted to the hospital with chief complaints of worsening dry cough and dyspnea on exertion of 1 month's duration. She was noted to have an abnormality on chest X-ray and was referred to our hospital for further examination. Contrast-enhanced chest computed tomography (CT) revealed a low-density tumor obstructing the left pulmonary artery (fig. 1a-d) and a pedunculated lesion extending to the main and right pulmonary arteries. A small part of the tumor also extended to the mediastinal space outside the pulmonary artery invading the left superior pulmonary vein, but there was no evidence of invasion of the trachea or bronchium. A fluorodeoxyglucose-positron emission tomography (FDG-PET) scan revealed a high uptake by the tumor of the pulmonary artery (max. standardized uptake value 23.11), multiple metastases in both lungs, bones (right 4th and 11th ribs, right ischial bone) and both adrenal glands (fig. 2). Suspected primary lesions other than that in the pulmonary artery were not identified. Tumor markers, including carcinoembryonic antigen, carbohydrate antigen (CA) 19-9, cytokeratin 19 fragment (CYFRA), pro-gastrin-releasing peptide, CA15-3, and CA125, were within the normal limits. No abnormal findings in the gastrointestinal tracts, gynecologic organs, and breasts were detected. Thus, the diagnosis of primary tumor of the pulmonary artery was made. Catheter angiography of the heart and pulmonary arteries revealed that blood flow to the left pulmonary artery was interrupted by the filling tumor and the fluttering pedunculated tumors extending from the main tumors into the trunk and right pulmonary arteries. 
Kumagai et al.: Successful Chemoradiotherapy for Undifferentiated Malignant Neoplasm Arising from the Left Pulmonary Artery

Small tumor samples could be obtained by catheter aspiration biopsy, but hemorrhagic cardiac tamponade occurred during the procedures, and drainage by pericardiocentesis and blood transfusion were urgently required. Histopathological examination showed a colonization of atypical small round cells of irregular shapes, hyperchromatic nuclei and eosinophilic cytoplasms. Tumor specimens were immunohistochemically positive for vimentin and partially positive for cytokeratin, but negative for S-100 protein, vascular endothelial markers [e.g., cluster of differentiation (CD)31, CD34, von Willebrand factor], myogenic markers (e.g., alpha-smooth muscle actin and muscle-specific actin, desmin), lymphocyte markers (e.g., CD45, CD20, CD79a, CD3), and lung cancer markers (e.g., thyroid transcription factor-1, surfactant protein A) (fig. 3). On the basis of the morphological and immunohistochemical findings, the tumor was finally diagnosed as an undifferentiated malignant neoplasm arising from the pulmonary artery. Anticoagulant therapy with warfarin was started for the prevention of thromboembolism as a result of tumor of the pulmonary artery, but the risk of sudden death due to thromboembolism remained high. Surgical resection of the pulmonary artery tumor and replacement with an artificial vessel was not employed, because this highly invasive surgery might have compromised the patient's general condition and because of the expected subsequent difficulties for further systemic therapy for the remaining metastatic lesions. Since local control of the intrapulmonary artery tumor was thought to be the prime determinant of short-term prognosis, chemoradiotherapy consisting of irradiation (50 Gy/25 fractions) of the pulmonary artery tumor and concurrent systemic chemotherapy with paclitaxel $\left(40 \mathrm{mg} / \mathrm{m}^{2}\right.$, administered on days 1,8 , $15,22,29$, and 36) and carboplatin (area under the curve 2, on the same days) were performed. According to the common terminology, criteria for adverse events version 3.0, grade 3 esophagitis, grade 2 thrombocytopenia, and grade 3 febrile neutropenia were observed, and chemotherapy was stopped on day 36. Radiation therapy was completed. Thirty-five days after the initiation of chemoradiotherapy, pulmonary artery tumor size had markedly decreased, an improvement of the perfusion in the left lung was seen (fig. 1e, f), and there was no sign of metastasis progression. The combination therapy of paclitaxel 175 $\mathrm{mg} / \mathrm{m}^{2}$, on day 1 , every 3 weeks) and carboplatin (area under the curve 5 , on day 1 , every 3 weeks) was subsequently continued for 5 months without tumor progression. However, the patient died of bleeding from the right adrenal metastatic lesion 9 months after the initial diagnosis.

\section{Discussion}

Tumors originating from the heart and large vessels are found at an incidence of about $0.02 \%$ at autopsy [4]. Tumors of the pulmonary artery are furthermore rare tumors; in fact, only approximately 200 cases have been reported in the literature. Since lethal events frequently occur in patients with pulmonary artery tumor when initial symptoms are present, $60 \%$ of these cases have previously been diagnosed by autopsy [5]. More recently, advances in imaging technologies have allowed more successful antemortem diagnosis [2]. To obtain sufficient tumor samples for histological diagnosis, open chest surgery under general anesthesia was required. Performing the appropriate therapy based on a definitive diagnosis was often difficult for such patients who were not suitable candidates for surgery. Tumor biopsy via intravenous catheterization has recently been used to obtain a small amount of tumor samples in such patients, as was done in the present case.

Most primary tumors of the pulmonary artery are sarcomas, including angiosarcoma, leiomyosarcoma, rhabdomyosarcoma, fibrosarcoma and unclassified undifferentiated 
Kumagai et al.: Successful Chemoradiotherapy for Undifferentiated Malignant Neoplasm Arising from the Left Pulmonary Artery

sarcoma [6-9]. Secondary/tertiary pulmonary artery tumors include those caused by direct invasion by lung cancer [10] and hematogenous metastases from hepatocellular carcinoma, renal cell carcinoma, breast cancer, and colorectal cancer [11]. Our patient had multiple tumor lesions in the bilateral lungs, bones, and adrenal glands. A diagnosis of primary pulmonary artery tumor was made, as that lesion was the largest and had the highest uptake of FDG compared to all the other lesions, which were more consistent with metastatic lesions. Although a small part of the tumor located in the mediastinal region suggested the possibility of direct invasion by a tumor outside the pulmonary artery, based on the clinical findings the tumor was more likely to arise from the pulmonary artery.

Histopathological findings in the tumor tissue from the intrapulmonary artery tumor included undifferentiated small atypical cells. Although immunohistochemical examination was intensively performed, it was difficult to identify the definitive origin of the tumor; ultimately, the lesion was diagnosed as an undifferentiated malignant neoplasm of pulmonary artery. In terms of therapies, surgery has been associated with favorable survival outcomes in a study of the primary pulmonary artery intimal sarcoma without distant metastasis. While overall survival of the patients treated without surgical operation was $11 \pm$ 3 months, that of the patients treated with surgical operation was $36.5 \pm 20.2$ months, and cases of long-term survival were observed [3]. Therefore, surgical resection of the pulmonary artery tumor in appropriate surgical candidates might be effective in reducing the risk of sudden death. Radiation therapy has also had mild antitumor effects and produced symptom relief in patients with these lesions who were not candidates for surgery [12]. Chemotherapies with doxorubicin-based regimens for sarcomas of the pulmonary artery have been utilized, but without objective response [12]. Specific regimens for pulmonary artery sarcoma have not been established, because no regimen has produced significant responses or benefits in terms of outcomes.

Cases of pulmonary artery tumor with metastasis were generally associated with poor outcomes with a median survival period of several months. Cardiopulmonary events were the major causes of death. Since our patient had undifferentiated malignant neoplasm obstructing the left pulmonary artery with multiple metastases, the therapeutic strategy should first provide local control, and any survival benefit likely requires good continuity of therapy. In most cases of chemotherapy for undifferentiated tumors arising from various organs, regimens for the differentiated tumor type of the corresponding organs are often employed. However, no effective chemotherapy has been reported for undifferentiated neoplasm of the pulmonary artery with distant metastasis, mostly because these cases are very rare. We selected combination chemotherapy with paclitaxel and carboplatin, referring to the regimens for carcinoma of unknown primary site [13]. Concurrent radiotherapy for the primary tumor was also performed based on the information from chemoradiotherapy regimens used against non-small cell lung cancer [14]. This therapy could achieve a significant reduction in the size of the tumors as well as symptomatic relief and long-term survival. In the present case, the patient's direct cause of death was hemorrhage from the right adrenal metastasis 9 months after the initial chemoradiotherapy, but no fatal events in conjunction with pulmonary arterial tumor were observed.

We conclude that chemoradiotherapy with carboplatin plus paclitaxel might be of benefit for patients with undifferentiated malignant neoplasm of the pulmonary artery who are not suitable candidates for surgery. Since this is a single instance of treatment of this disease, additional cases need to be collected, the use of multidisciplinary therapy needs to be assessed, and further biological analyses of undifferentiated neoplasm arising from the pulmonary artery need to be conducted. 


\begin{tabular}{l|l}
\hline DOI: $10.1159 / 000365387$ & C 2014 S. Karger AG, Basel
\end{tabular}

Kumagai et al.: Successful Chemoradiotherapy for Undifferentiated Malignant Neoplasm Arising from the Left Pulmonary Artery

\section{Disclosure Statement}

The authors declare no conflicts of interest associated with this paper.

\section{References}

1 Roberts KE, Hamele-Bena D, Saqi A, Stein CA, Cole RP: Pulmonary tumor embolism: a review of the literature. Am J Med 2003;115:228-232.

$>2$ Restrepo CS, Betancourt SL, Martinez-Jimenez S, Gutierrez FR: Tumors of the pulmonary artery and veins. Semin Ultrasound CT MR 2012;33:580-590.

-3 Blackmon SH, Rice DC, Correa AM, Mehran R, Putnam JB, Smythe WR, Walkes JC, Walsh GL, Moran C, Singh H, Vaporciyan AA, Reardon M: Management of primary pulmonary artery sarcomas. Ann Thorac Surg 2009;87:977-984.

$\checkmark 4$ Amano J, Nakayama J, Yoshimura Y, Ikeda U: Clinical classification of cardiovascular tumors and tumor-like lesions, and its incidences. Gen Thorac Cardiovasc Surg 2013;61:435-447.

$>5$ Ramp U, Gerharz CD, Iversen S, Schweden F, Steppling H, Gabbert HE: Sarcoma of the pulmonary artery: report of 2 cases and a review of the literature. J Cancer Res Clin Oncol 1992;118:551-556.

-6 Burke AP and Virmani R: Sarcomas of the great vessels. A clinicopathologic study. Cancer 1993;71:17611773.

$>7$ Cox JE, Chiles C, Aquino SL, Savage P, Oaks T: Pulmonary artery sarcoma: a review of clinical and radiologic features. J Comput Assist Tomogr 1997;21:750-755.

-8 Nonomura A, Kurumaya H, Kono N, Nakanuma Y, Ohta G, Terahata S, Matsubara F, Matsuda T, Asaka T, Nishino T: Primary pulmonary artery sarcoma: report of two autopsy cases studied by immunohistochemistry and electron microscopy, and review of 110 cases reported in the literature. Acta Pathol Jpn 1988;38:883-896.

-9 Gaumann A, Bode-Lesniewska B, Zimmermann DR, Fanburg-Smith JC, Kirkpatrick CJ, Hofstädter F, Woenckhaus M, Stoehr R, Obermann EC, Dietmaier W, Hartmann A: Exploration of the APC/beta-catenin (WNT) pathway and a histologic classification system for pulmonary artery intimal sarcoma. A study of 18 cases. Virchows Arch 2008;453:473-484.

10 Kolin A, Koutoulakis T: Invasion of pulmonary arteries by bronchial carcinomas. Hum Pathol 1987;18:1165-1167.

11 Roberts KE, Hamele-Bena D, Saqi A, Stein CA, Cole RP: Pulmonary tumor embolism: a review of the literature. Am J Med 2003;115:228-232.

-12 Penel N, Taieb S, Ceugnart L, Dansin E, Hoguet D, Vanseymortier L, Lartigau E: Report of eight recent cases of locally advanced primary pulmonary artery sarcomas: failure of doxorubicin-based chemotherapy. J Thorac Oncol 2008;3:907-911.

13 Huebner G, Link H, Kohne CH, Stahl M, Kretzschmar A, Steinbach S, Folprecht G, Bernhard H, Al-Batran SE, Schoffski P, Burkart C, Kullmann F, Otremba B, Menges M, Hoffmann M, Kaiser U, Aldaoud A, Jahn A; German CUP Study Group: Paclitaxel and carboplatin vs gemcitabine and vinorelbine in patients with adeno- or undifferentiated carcinoma of unknown primary: a randomised prospective phase II trial. Br J Cancer 2009;100:44-49.

14 Yamamoto N, Nakagawa K, Nishimura Y, Tsujino K, Satouchi M, Kudo S, Hida T, Kawahara M, Takeda K, Katakami N, Sawa T, Yokota S, Seto T, Imamura F, Saka H, Iwamoto Y, Semba H, Chiba Y, Uejima H, Fukuoka M: Phase III study comparing second- and third-generation regimens with concurrent thoracic radiotherapy in patients with unresectable stage III non-small-cell lung cancer: West Japan Thoracic Oncology Group WJTOG0105. J Clin Oncol 2010;28:3739-3745. 
Kumagai et al.: Successful Chemoradiotherapy for Undifferentiated Malignant Neoplasm Arising from the Left Pulmonary Artery

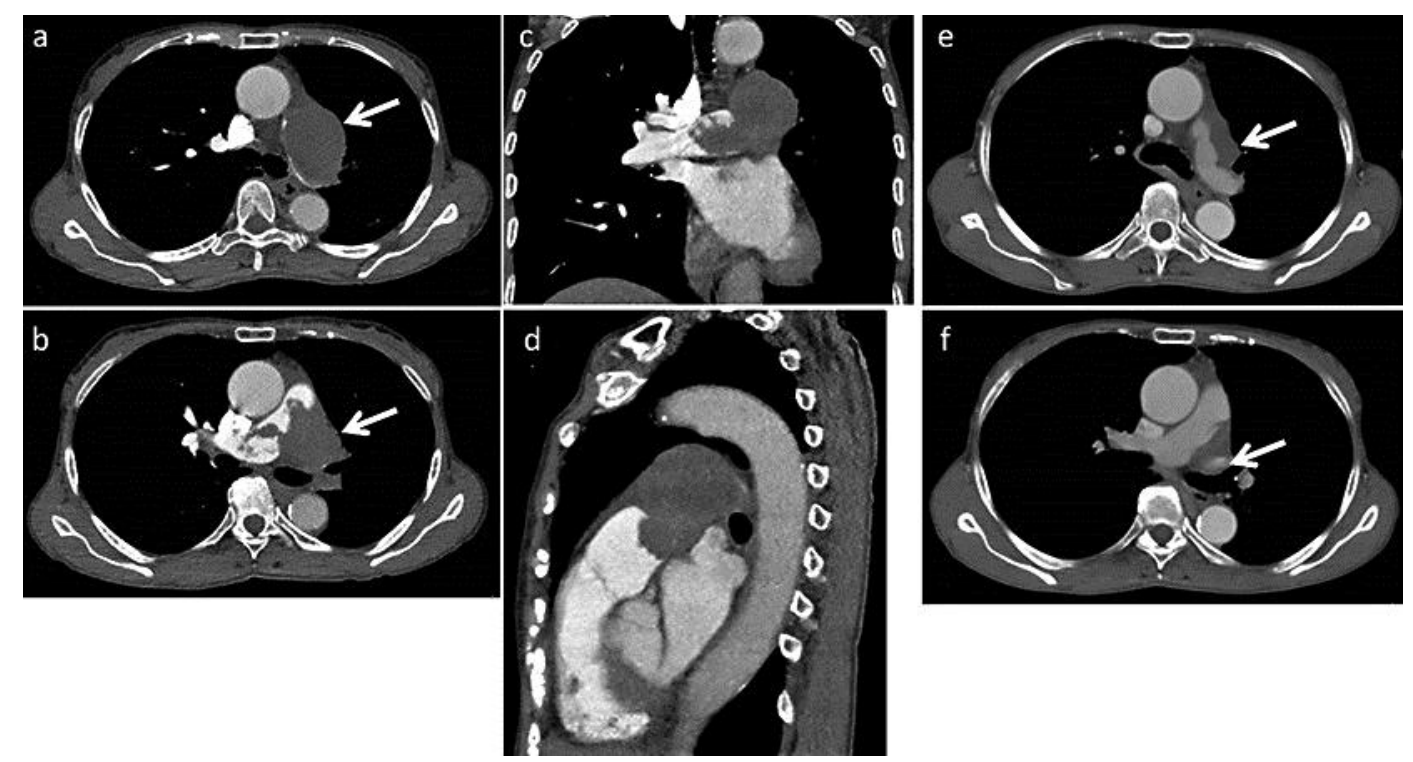

Fig. 1. Contrast-enhancedCT images of the pulmonary artery tumor. Axial (a, b), coronal (c), and sagittal (d) images at the time of initial diagnosis are shown. CT after chemoradiotherapy (e, f) shows a contraction of the intrapulmonary artery tumor and consecutive mediastinal mass region. Arrows indicate the pulmonary artery tumor.

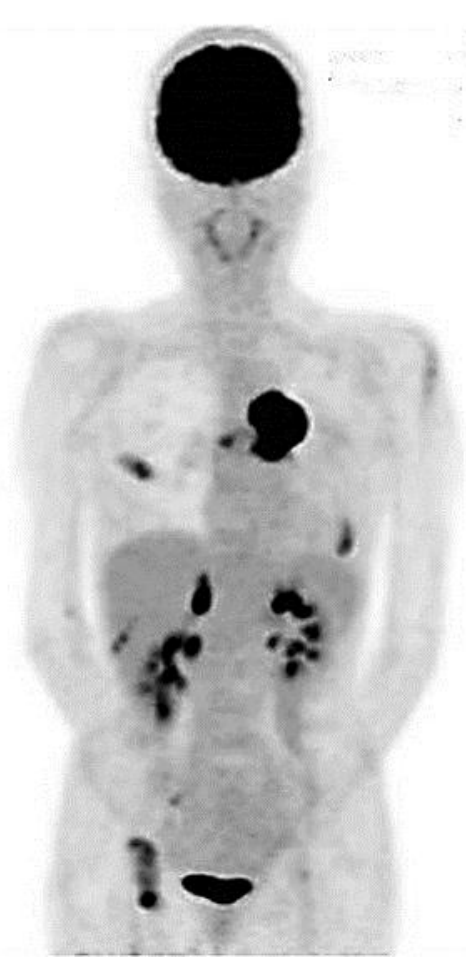

Fig. 2. FDG-PET scan at the time of initial diagnosis. The tumor of the pulmonary artery showed high FDG uptake (max. standardized uptake value 23.11). Multiple metastases in both lungs, bones, and both adrenal glands were detected. 


\section{Case Reports in Oncology}

\begin{tabular}{l|l}
\hline Case Rep Oncol 2014;7:484-490 \\
\hline DOI: $10.1159 / 000365387$ & $\begin{array}{l}\text { C 2014 S. Karger AG, Basel } \\
\text { www.karger.com/cro }\end{array}$ \\
\hline
\end{tabular}

Kumagai et al.: Successful Chemoradiotherapy for Undifferentiated Malignant Neoplasm Arising from the Left Pulmonary Artery
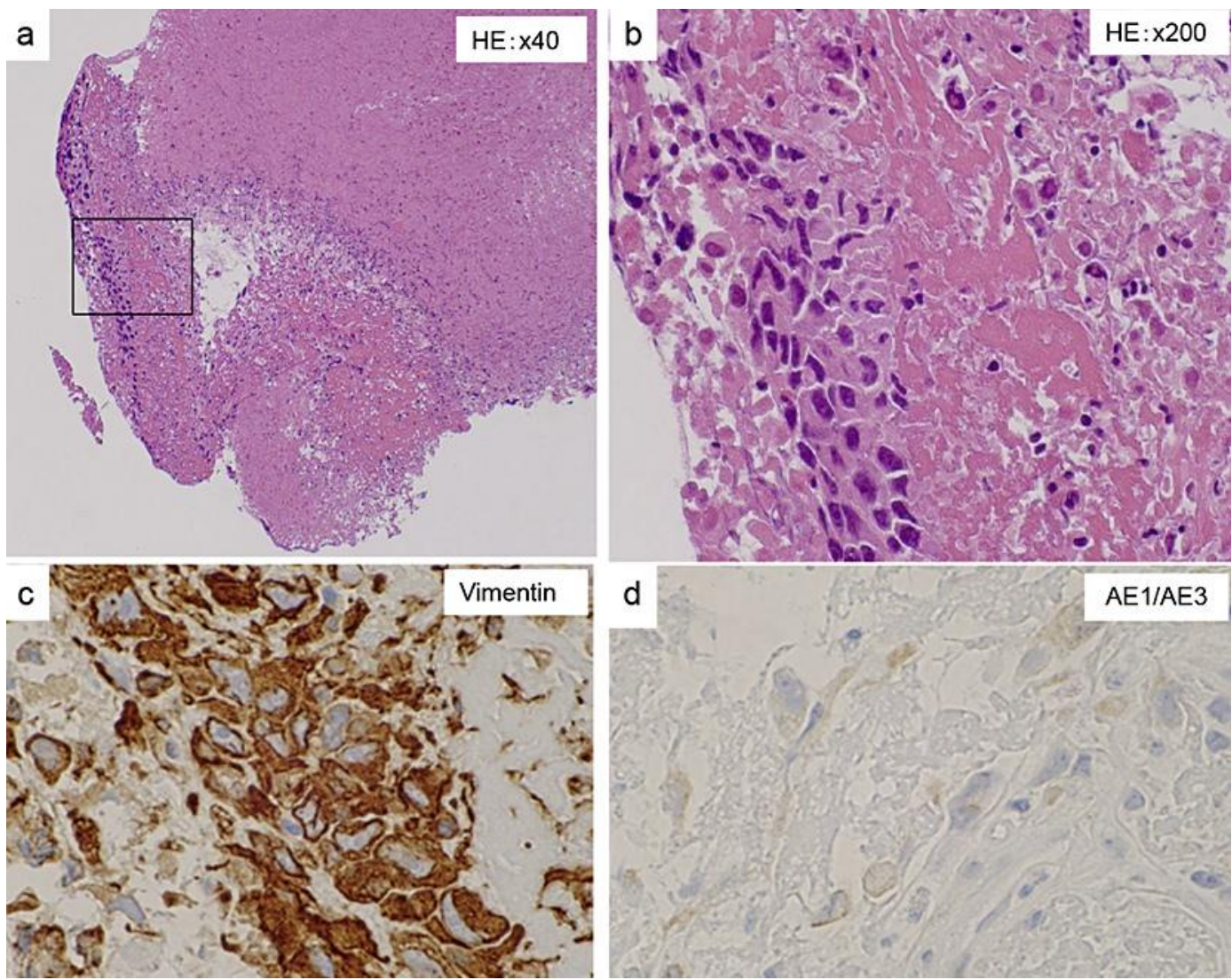

Fig. 3. Histopathological examination of the pulmonary artery tumor specimen obtained by catheter aspiration biopsy. a Tumor cells associated with necrotic tissue. HE. $\times 40$. $\mathbf{b}$ Tumor cells stained by HE. $\times 200$. c Tumor cells were positively stained by anti-vimentin antibody. $\times 200$. $\mathbf{d}$ Tumor cells were focally positive in the staining by anti-AE1/AE3 antibody. $\times 200$. 